\title{
Robust auction design under multiple priors by linear and integer programming
}

\author{
Çağıl Koçyiğit ${ }^{2}$ • Halil I. Bayrak ${ }^{1}$ • Mustafa Ç. Pınar ${ }^{1}$
}

Published online: 17 February 2017

(C) Springer Science+Business Media New York 2017

\begin{abstract}
It is commonly assumed in the optimal auction design literature that valuations of buyers are independently drawn from a unique distribution. In this paper we study auctions under ambiguity, that is, in an environment where valuation distribution is uncertain itself, and present a linear programming approach to robust auction design problem with a discrete type space. We develop an algorithm that gives the optimal solution to the problem under certain assumptions when the seller is ambiguity averse with a finite prior set $\mathcal{P}$ and the buyers are ambiguity neutral with a prior $f \in \mathcal{P}$. We also consider the case where all parties, the buyers and the seller, are ambiguity averse, and formulate this problem as a mixed integer programming problem. Then, we propose a hybrid algorithm that enables to compute an optimal solution for the problem in reduced time.
\end{abstract}

Keywords Optimal auction design $\cdot$ Robustness $\cdot$ Multiple priors $\cdot$ Ambiguity $\cdot$ Linear programming $\cdot$ Mixed-integer programming

\section{Introduction}

An auction is a process of selling a single/multiple good(s). Auctions have been used since antiquity for selling a variety of goods. They continue to be popular not only for the sale of art objects but also for the sale of goods as varied as fish, tobacco, flowers and so on. Auctions are also used in competitive bidding for procurement in several industries where the bidders now try to sell their goods instead of acquiring something. Auctions have also been the preferred method in transferring the ownership or usage rights of public goods such as frequency spectrum to private hands. Therefore, determining the most profitable auction

Mustafa Ç. Pınar

mustafap@bilkent.edu.tr

1 Department of Industrial Engineering, Bilkent University, 06800 Ankara, Turkey

2 Ecole Polytechnique Federale de Lausanne, Lausanne, Switzerland 
rule in a given context is a crucial research question of interest to both the public and private sectors (Klemperer 1999).

A common aspect of auctions is the collection of bids from buyers. An auction is described by an allocation rule specifying who gets the object and a payment rule describing how much every bidder must pay. In auctions, each buyer has a valuation — willingness to pay-assigned to goods on sale. The major reason for holding auctions is the seller's lack of knowledge about these valuations. Hence, the question is determine the rules of allocation and payment (e.g., in a sealed bid auction, the highest bidder wins and pays the second highest bid amount) that are optimal with respect to some suitable criteria (e.g., maximizing the expected revenue of the seller) for the party running the auction while ensuring by appropriate incentives the participation of bidders into the process. This endeavour is referred to as "auction design", i.e., it indicates the design of the auction process. In optimal auction design literature, it is mostly assumed that buyers' valuations are independently drawn from a unique distribution. However, in reality, it is more likely that some estimation errors occur or that one has no clear prior idea of the valuations of potential bidders, and thus, attaching a precise distribution to this valuation is a questionable approach, if not impossible. Therefore, it is a worthwhile research effort to optimally design auctions taking into account the uncertainty in the valuation distribution of bidders. This line of research is henceforth referred to as robust auction design in the sense that the resulting auction rules are robust against uncertainty in the valuation distribution which is also termed ambiguity in the economics literature. Robustness in this context is to yield expected revenue figures that are stable regardless of which distribution the valuations are drawn from.

In this paper, we study auctions in an environment where valuation distribution comes from a set $\mathcal{P}$ of possible distributions, and introduce a linear programming approach to robust auction design problem where a single object is sold to potential buyers. To have a finite number of equations in our formulation and to take advantage of advances in modern optimization tools, we let the valuation distribution to be discrete as well as the set $\mathcal{P}$. In the literature, it is shown that the decision makers may exhibit some degree of ambiguity averse behavior (Ellsberg 1961). Here we consider the seller to be ambiguity averse in the sense that she tries to maximize the worst case expected revenue. Hence, we adopt a more realistic approach to formulate auction design problems compared to the studies with unique valuation distribution assumption.

This paper is organized as follows: Sect. 2 provides a brief literature review on auction design. Some important concepts related to our study are introduced. In Sect. 3 we define robust auction design problem when the seller is ambiguity averse and the buyers are ambiguity neutral. Note that ambiguity neutrality of buyers leads them to give the same importance to all possible realizations of the valuation distribution. We reformulate this problem as a linear programming problem. Then, we develop a simple procedure which gives the optimal solution under certain assumptions and state properties of the optimal mechanism. In Sect. 4 we introduce the robust auction design problem when the buyers are ambiguity averse too. We give a reformulation of the problem as a mixed integer programming problem. Since the optimal solution does not result in a recognizable mechanism we focus on efficient numerical solution of problem instances. To this end, we propose an efficient algorithm. We support our claim by computational results. Finally, we give concluding remarks in Sect. 5.

Contributions of this paper are as follows:

1. In Sect. 3, we give a specific and applicable optimal mechanism for the robust auction design problem with ambiguity averse seller and ambiguity neutral buyers under certain assumptions, which is the only detailed optimal mechanism in the literature to our 
knowledge. Our optimal mechanism is easy to understand due to its similarity to the well-known Vickrey auction, and it is reasonable and fair from participants' perspective because only the winner makes a payment which never exceeds his own bid.

2. In Sect. 4, the MIP formulation is new, to the best of our knowledge, as well as the algorithm. The contribution here is to render the robust auction design problem with ambiguity averse seller and buyers tractable in that it is solvable by existing state-ofthe-art optimization solvers. To shorten the solution time, we propose an algorithm and demonstrate its usefulness by computational results.

\section{Literature review}

In this section, we give a brief literature review related to our work. For a more detailed review, see Klemperer (1999). We also recommend (Krishna 2009) as an introductory book. Since auction design can be considered as a sub-branch of economic mechanism design, we refer to the general reference (Hurwicz and Reiter 2006) on economic mechanism design.

Auction design entered the economics literature relatively recently. Vickrey (1961) wrote the first game theoretical analysis of auctions. This was the first occurrence of well-known second price sealed-bid auctions in which buyers simultaneously report sealed bids to the seller, the highest bidder wins the object and pays the second highest bid. Today, second price sealed-bid auctions are also called Vickrey auctions.

Myerson (1981) stated the Revelation Principle:

\section{The outcomes resulting from any equilibrium of any mechanism can be replicated by} a truthful equilibrium of some direct mechanism.

By the Revelation Principle, Myerson (1981) concluded that restricting attention to only direct mechanisms, i.e., mechanisms where all the buyers report their true valuations, does not cause loss of generality under certain assumptions. Utilizing this result, he also showed that the second price auction with a reserve price is an optimal mechanism to classical auction design problem when the hazard function defined as the ratio of density function to survival function (one minus cumulative distribution function), is monotone (Myerson 1981). In classical auction design problem, there is a risk neutral seller with a single good which she desires to sell to a number of risk neutral buyers. Each buyer has a private valuation assigned to the good. Buyers' valuations are assumed to be independently drawn with respect to a unique continuous distribution function over a finite interval.

In 1981, simultaneously, Myerson (1981), and Riley and Samuelson (1981) extended Vickrey's results regarding expected revenue equivalence in different auctions and led to the famous Revenue Equivalence Principle:

Under certain conditions, any auction mechanism that results in identical outcomes

(i.e. allocates items to the same bidders) also generates the same expected revenue.

Myerson (1981) also analyzed optimal auctions when the monotone hazard function and symmetric buyers assumptions are relaxed.

When risk aversion is introduced to the auction design problems, the Revelation Principle is not valid for most of the cases. For analyses of how risk aversion affects the Revelation Principle and literature in risk aversion, we direct the reader to Klemperer (1999). In this paper, we assume that the seller and the buyers are risk neutral. 
Recently, Vohra (2012) showed the close relationship between linear programming and auction design when valuations of buyers are discrete. He used standard results from linear programming to solve a wide class of auction design problems. His work has been a motivation for the present paper to use linear programming in robust auction design problem. Furthermore, although auction problems have been widely studied in the literature, results on robust auction design are limited due to the complexity of the problem. In Gilboa and Schmeidler (1989) modeled ambiguity aversion using maxmin expected utility (MMEU). In MMEU, decision maker is characterized by a utility function and a set of priors and the chosen act maximizes the minimal expected utility over the prior set. In this paper, we follow their work to formulate robust auction design problem. There have been few studies on auction design allowing ambiguity in prior distribution. Most of these studies consider some specific auctions, such as first price auction and second price auction, rather than seeking an optimal auction (Salo and Weber 1995; Lo 1998). Bandi and Bertsimas (2014) studied optimal design for multi-item auction from a robust optimization perspective but this study is quite different from our work. Rather than specifying an ambiguity set for the type distribution as done here, they treat the buyer valuations as uncertain parameters which are allowed to take values in some uncertainty sets designed to reflect the usual probability axioms in a limiting sense in an auction setting with a reservation price.

Bose et al. (2006) is closer to our work. However, there are marked differences between Bose et al. (2006) and our work. The first difference from our approach is that the valuation distribution $f$ is assumed to be continuous over a finite interval and the prior set $\mathcal{P}$ is infinite in Bose et al. (2006). Besides, our incentive compatibility constraints in Sect. 3 under multiple priors are different from theirs. This is because when Bose et al. (2006) considers ambiguity neutral agents, it is assumed that those agents have a unique prior. In our setting, we consider the problem from the sellers' perspective and he does not have this information. Instead of eliminating ambiguity, we assume that ambiguity neutral agents stick with linear utility functions for each distribution from the prior set instead of switching to MMEU. The important trick is to find a mechanism which is incentive compatible for all distributions in the prior set since each buyer may have different distributions as their prior. Under monotone hazard function assumption, in Bose et al. (2006) it is proved that when the seller is ambiguity averse and the bidders are ambiguity neutral, an auction that fully insures the seller is in the set of optimal mechanisms. The theorem and proof for this result are based on the assumption that buyers have a unique prior; hence, an insurance mechanism is not optimal in our setting. In Sect. 3, we derive an optimal mechanism for robust auction design problem and claim that this is the unique optimal mechanism. Furthermore, since we work in a discrete type space and our formulations are linear and integer optimization formulations we are able to harness the power of modern optimization tools, which is a feature absent from Bose et al. (2006).

Under certain assumptions some properties of optimal mechanism were given in Bose et al. (2006) when buyers are also ambiguity averse. Bose et al. (2006) showed that when the bidders face more ambiguity than the seller in a way that buyers' prior set contains the seller's prior set, the seller can be better off by switching to an auction providing full insurance to all types of bidders, ${ }^{1}$ and in general neither the first nor the second price auction is optimal.

1 A full insurance mechanism is one where the ex-post pay-off of a given type of bidder does not vary with the report of a competing bidder. 


\section{Auction design problem with ambiguity averse seller}

In our problem environment, an agent knows his own valuation, and he also believes that others' valuations are independently drawn from a finite and discrete type set $T=\{1, \ldots, m\}$ with respect to a probability mass function $f$ satisfying $f_{i}>0$ for all $i \in T$. The seller is not sure about the maximum amount each buyer is willing to pay for the object, which we call valuation (type) of agent. On the other hand, the seller wishes to protect herself against uncertainty in the distribution of buyer valuations by specifying a discrete prior set $\mathcal{P}$ with a finite number of distributions in it. Therefore, we have a single, ambiguity averse seller with prior set $\mathcal{P}$ and $n$ ambiguity neutral buyers (agents). Both the seller and the agents are risk neutral. In other words, they have linear utility functions.

The seller desires to sell a single good to the agents. Since the seller is ambiguity averse, the objective is to maximize her worst case expected revenue. To formulate this problem, we invoke the Revelation Principle (which also holds in our case; see Bose et al. 2006), and restrict our attention only to direct mechanisms in which agents simultaneously report their true valuations. From Sect. 2, recall that the Revelation Principle states that the outcomes resulting from any equilibrium of any mechanism can be replicated by a truthful equilibrium of some direct mechanism.

\subsection{Formulation}

Before problem formulation, let us give the notation. We use $t \in T^{n}$ to denote a profile vector which is constructed by reports of all agents. The symbols $a$ and $p$ are defined to be allocation and payment rule, respectively.

For an indivisible object, fractional values of continuous allocation rule variables are interpreted as the probability of a bidder getting the object. Obviously, in case the object is divisible, fractional allocation values refer to the fraction of the good. The symmetry assumption allows focusing on one agent, say agent 1 . Therefore, we let $a\left(i, t^{-1}\right)$ be the allocation to agent 1 and $p\left(i, t^{-1}\right)$ be the payment done by agent 1 to the seller when he reports his type as $i \in T$ and all other agents report $t^{-1} \in T^{n-1}$. We will also use them as $a_{i}(t)$ and $p_{i}(t)$, allocation and payment of agent who reported type $i \in T$ in profile $t \in T^{n}$. The probability of agents having types that give rise to the profile $t^{-1}$ is denoted by $\pi_{f}\left(t^{-1}\right)$ for all $f \in \mathcal{P}$. The number of agents with type $i$ in profile $t$ is shown by $n_{i}(t)$.

Interim (expected) allocations and payments are denoted accordingly:

$$
\begin{aligned}
& A_{f}(i)=\sum_{t^{-1} \in T^{n-1}} a_{i}\left(i, t^{-1}\right) \pi_{f}\left(t^{-1}\right) \quad \forall f \in \mathcal{P}, \\
& P_{f}(i)=\sum_{t^{-1} \in T^{n-1}} p_{i}\left(i, t^{-1}\right) \pi_{f}\left(t^{-1}\right) \quad \forall f \in \mathcal{P} .
\end{aligned}
$$

To clarify, $A_{f}(i)$ denotes expected allocation to agent 1 and $P_{f}(i)$ is the payment of agent 1 if he reports type $i$ where $f \in \mathcal{P}$. The seller faces the following constrained maximization problem $\left(o p t^{1}\right)$ over the variables $A_{f}(i), P_{f}(i)$, and $a_{i}(t)$ :

$$
\begin{array}{llrl}
\max _{A, P, a} & \left\{\min _{f \in \mathcal{P}} \sum_{i \in T} f_{i} P_{f}(i)\right\}, & \\
\text { s.t. } & i A_{f}(i)-P_{f}(i) \geq i A_{f}(j)-P_{f}(j) & \forall i, j \in T & \forall f \in \mathcal{P}, \\
& i A_{f}(i)-P_{f}(i) \geq 0 & \forall i \in T & \forall f \in \mathcal{P}, \\
& A_{f}(i)=\sum_{t^{-1} \in T^{n-1}} a_{i}\left(i, t^{-1}\right) \pi_{f}\left(t^{-1}\right) & \forall i \in T & \forall f \in \mathcal{P},
\end{array}
$$




$$
\begin{array}{lr}
\sum_{i \in T} n_{i}(t) a_{i}(t) \leq 1 & \forall t \in T^{n}, \\
a_{i}(t) \geq 0 & \forall i \in T, \forall t \in T^{n} .
\end{array}
$$

The objective is to maximize the seller's worst case expected revenue (1). I.e., since the seller does not know which member of $\mathcal{P}$ is the true valuation distribution function, she tries to maximize the minimum expected revenue over $f \in \mathcal{P}$ due to ambiguity aversion. Bidders are utility maximizers such that, given a mechanism, a bidder with true valuation $i$ tries to maximize $i A_{f}(j)-P_{f}(j)$ over $j$. Constraints (2) are called Bayes-Nash Incentive Compatibility (BNIC) constraints in the literature. These constraints ensure that, for an agent, misreporting the valuation will always result in expected utility which is less than or equal to the one when the type is truthfully reported. Note that we are only interested in direct mechanisms and, by BNIC, a risk neutral agent's optimal strategy is to truthfully report his valuation. With constraints (3), each agent will choose to participate in the auction because he will gain a non-negative expected payoff in every possible outcome of profiles. This type of constraints is known as Individual Rationality (IR) constraints. Constraints (4) satisfy the consistency between interim allocations and allocation rule variables. Obviously, constraints (5) and (6) ensure that at most one good is allocated (whole or in part) for each profile outcome and no agent receives a negative amount. Next, we associate shortest path problems with BNIC and IR constraints to reformulate $\left(o p t^{1}\right)$.

\subsection{Network representation}

In this section, we follow Vohra's approach (2012), and relate to shortest path problems and duality theory. Consider (2) and (3). They can be rewritten as follows:

$$
\begin{gathered}
i A_{f}(i)-i A_{f}(j) \geq P_{f}(i)-P_{f}(j) \quad \forall i, j \in T \quad \forall f \in \mathcal{P}, \\
i A_{f}(i) \geq P_{f}(i) \quad \forall i \in T \quad \forall f \in \mathcal{P} .
\end{gathered}
$$

For each $f \in \mathcal{P}$, we can associate system (2) and (3) with the following network:

In Fig. 1, each vertex corresponds to a type in $T$. A dummy type with value 0 -with $A_{f}(0)$ and $P_{f}(0)$ equal to 0 for all $f \in \mathcal{P}$-is introduced to the network to include IR constraints (3.3) to the network representation. There is a directed edge of length $i A_{f}(i)-i A_{f}(j)$ between every ordered pair of types $(j, i)$.

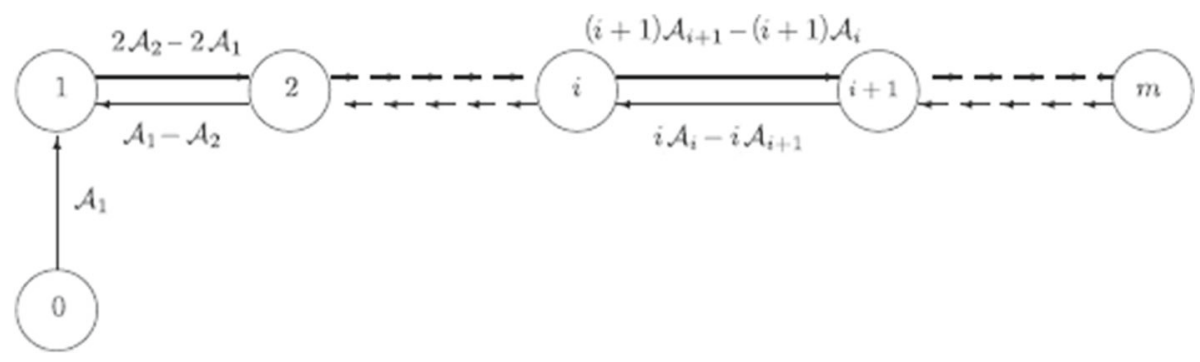

Fig. 1 Network of valuations 
Now, consider the following shortest path problem from vertex 0 to vertex $m$ :

$$
\begin{array}{ll}
\min & \sum_{i \in T} \sum_{j \in T}\left(i A_{f}(i)-i A_{f}(j)\right) x_{j i} \\
\text { s.t. } & \sum_{j \in T} x_{j i}-\sum_{j \in T} x_{i j}= \begin{cases}1 & \text { if } i=m \\
-1 & \text { if } i=0 \\
0 & \text { otherwise }\end{cases} \\
& x_{i j} \in\{0,1\} \quad \forall i, j \in T .
\end{array}
$$

We can let $x_{i j}$ 's take continuous values, and the optimal solution to the relaxed shortest path problem will still be an integer solution due to the total unimodularity property of the feasible set. Note that we consider the relaxed shortest path problem from this point onwards.

For fixed interim allocation values, if we interpret $P_{f}(i)$ 's to be dual variables corresponding to each constraint of the shortest path problem then we observe that (2) and (3) are the constraints of the dual problem. Hence, system (2) and (3) is feasible if and only if the network has no negative length cycles. Otherwise, the shortest path problem is unbounded, which leads the corresponding dual problem to be infeasible.

Theorem 1 The system (2)-(3) is feasible if and only if interim allocations are monotonic, i.e., if $i \leq j$, then $A_{f}(i) \leq A_{f}(j)$ for all $f \in \mathcal{P}$.

For a proof, see Vohra (2012). Note that to avoid negative length cycles, the length of the edge from $i$ to $i+2$ must be at least as large as the sum of the lengths of edges $(i, i+1)$ and $(i+1, i+2)$. This implies that Fig. 1 includes all shortest paths from vertex 0 to $m$. We also observe that in absence of negative cycles, the shortest path from vertex 0 to $i$ gives the tightest upper bound for each $P_{f}(i)$. Since the objective is to maximize sum of $P_{f}(i)$ 's with non-negative coefficients, it is reasonable to set them equal to their tightest upper bounds. Therefore, we can rewrite the objective as follows:

$$
\begin{aligned}
\sum_{i \in T} f_{i} P_{f}(i) & =\sum_{i \in T} f_{i} \sum_{k=1}^{i} k A_{f}(k)-k A_{f}(k-1)=\sum_{i \in T} f_{i}\left(i A_{f}(i)-\sum_{k=1}^{i} A_{f}(k-1)\right) \\
& =\sum_{i \in T} f_{i} i A_{f}(i)-(1-F(i)) A_{f}(i)=\sum_{i \in T} f_{i}\left(i-\frac{1-F(i)}{f_{i}}\right) A_{f}(i) .
\end{aligned}
$$

We let $v_{f}(i)=i-\frac{1-F(i)}{f_{i}}$. Using the development so far, $\left(o p t^{1}\right)$ can be reformulated as follows:

$$
\begin{aligned}
& \max _{A, a}\left\{\min _{f \in \mathcal{P}} \sum_{i \in T} f_{i} v_{f}(i) A_{f}(i)\right\}, \\
& \text { s.t. } \quad 0 \leq A_{f}(1) \leq \cdots \leq A_{f}(m) \\
& \forall f \in \mathcal{P}, \\
& A_{f}(i)=\sum_{t^{-1} \in T^{n-1}} a_{i}\left(i, t^{-1}\right) \pi_{f}\left(t^{-1}\right) \quad \forall i \in T \quad \forall f \in \mathcal{P}, \\
& \sum_{i \in T} n_{i}(t) a_{i}(t) \leq 1 \quad \forall t \in T^{n}, \\
& a_{i}(t) \geq 0 \quad \forall i \in T, \forall t \in T^{n} .
\end{aligned}
$$

While the objective function takes a new form in (7), monotonicity of expected allocations (8) replaces BNIC (2) and IR (3). Vohra's (2012) next step is to take out allocation rule 
variables and solve the problem only over interim allocations. However, we will take out interim allocations instead because otherwise, we are unable to find a useful formulation to ensure existence of a corresponding allocation rule.

\subsection{Projecting out expected allocations}

We shall proceed as Vohra (2012), and show that his reformulation does not ensure feasibility of expected allocations in our problem. Vohra uses the following theorem to reduce the auction design problem without ambiguity to a polymatroid optimization problem.

Theorem 2 Border's Theorem Vohra (2012) The expected allocation A(i) is feasible if and only if

$$
n \sum_{i \in S} f_{i} A(i) \leq 1-\left(\sum_{i \notin S} f_{i}\right)^{n} \quad \forall S \subseteq T
$$

The proof follows from reformulating (4)-(6) as a transportation problem and standard maxflow-mincut characterization of feasibility (Vohra 2011). Note that in Vohra's problem definition, it is assumed that buyers' valuations depend on a unique distribution function. Hence, (4)-(6) refer to only one $f$.

In our formulation, since expected allocations differ for each $f \in \mathcal{P}$, we need to write inequalities from Border's theorem for all distributions:

$$
\begin{array}{ll}
\max _{A} & \left\{\min _{f \in \mathcal{P}} \sum_{i \in T} f_{i} v_{f}(i) A_{f}(i)\right\}, \\
\text { s.t. } \quad & 0 \leq A_{f}(1) \leq \cdots \leq A_{f}(m) \quad \forall f \in \mathcal{P} \\
& n \sum_{i \in S} f_{i} A_{f}(i) \leq 1-\left(\sum_{i \notin S} f_{i}\right)^{n} \quad \forall S \subseteq T \quad \forall f \in \mathcal{P} .
\end{array}
$$

This formulation decomposes for each $f \in \mathcal{P}$. The solutions from the decomposed problems will yield several allocation rules which may not be implementable at the same time. Hence, this approach is not suitable for our problem of maximizing the minimum expected revenue.

\subsection{Final form of the formulation}

We take out expected allocation variables and reformulate the problem accordingly:

$$
\begin{aligned}
\max _{a} & \left\{\min _{f \in \mathcal{P}} \sum_{i \in T} f_{i} v_{f}(i) \sum_{t^{-1} \in T^{n-1}} a_{i}\left(i, t^{-1}\right) \pi_{f}\left(t^{-1}\right)\right\}, \\
\text { s.t. } & 0 \leq \sum_{t^{-1} \in T^{n-1}} a_{1}\left(1, t^{-1}\right) \pi_{f}\left(t^{-1}\right) \leq \cdots \leq \sum_{t^{-1} \in T^{n-1}} a_{m}\left(m, t^{-1}\right) \pi_{f}\left(t^{-1}\right) \quad \forall f \in \mathcal{P} \\
& \sum_{i \in T} n_{i}(t) a_{i}(t) \leq 1 \quad \forall t \in T^{n} \\
& a_{i}(t) \geq 0 \quad \forall i \in T, \quad \forall t \in T^{n} .
\end{aligned}
$$


Introducing a new variable $z$, we can linearize this problem. Below, the final form of the formulation can be found.

$$
\begin{array}{ll}
\max _{a, z} & z, \\
\text { s.t. } & z \leq \sum_{i \in T} f_{i} \sum_{t^{-1} \in T^{n-1}} v_{f}(i) a_{i}\left(i, t^{-1}\right) \pi_{f}\left(i, t^{-1}\right) \quad \forall f \in \mathcal{P} \\
& \leq \sum_{t^{-1} \in T^{n-1}} a_{1}\left(1, t^{-1}\right) \pi_{f}\left(t^{-1}\right) \leq \cdots \\
& \leq \sum_{t^{-1} \in T^{n-1}} a_{m}\left(m, t^{-1}\right) \pi_{f}\left(t^{-1}\right) \quad \forall f \in \mathcal{P} \\
& \sum_{i \in T} n_{i}(t) a_{i}(t) \leq 1 \quad \forall t \in T^{n} \\
& a_{i}(t) \geq 0 \quad \forall i \in T, \quad \forall t \in T^{n} .
\end{array}
$$

This is a linear programming problem. Hence, it is easy to solve numerically using state-ofthe-art optimization software.

\subsection{The solution approach}

In this section, we first give Propositions 1, 2 and 3 representing some basic results concerning the allocation rule. Theorems 3, 4 and 5 clarify the cases in which it is optimal for ambiguity averse buyer to stick with Second Price Auction. For other cases, we propose an algorithm that constructs an optimal mechanism similar to Second Price Auction.

To derive an optimal mechanism from our final formulation, we focus on the case where there are two agents and the type distribution set is equal to $\mathcal{P}=\{f, g\}$. We also assume that the monotone hazard condition holds, which leads $v(i)$ to be non-decreasing in $i \in T$. If we ignore monotonicity of interim allocations (11), the two propositions below and results stated in between hold.

Proposition 1 Optimal allocation rule satisfies $a_{i}^{*}(i, j) \geq a_{j}^{*}(j, i), \forall(i, j) \in T^{2}$ such that $i \geq j$.

Proof We establish the result by analyzing coefficients of $a_{i}(i, j)$ and $a_{j}(j, i)$ in the objective function:

We aim to maximize $z$ such that

$$
\begin{aligned}
& z \leq \sum_{i \in T} \sum_{j \in T} f_{i} a_{i}(i, j) v_{f}(i) f_{j}, \\
& z \leq \sum_{i \in T} \sum_{j \in T} g_{i} a_{i}(i, j) v_{g}(i) g_{j} .
\end{aligned}
$$

For arbitrary $i$ and $j,(14)$ and (15) can be rewritten as

$$
\begin{aligned}
& z \leq \cdots+f_{i} f_{j} a_{i}(i, j) v_{f}(i)+f_{i} f_{j} a_{j}(j, i) v_{f}(j), \\
& z \leq \cdots+g_{i} g_{j} a_{i}(i, j) v_{g}(i)+g_{i} g_{j} a_{j}(j, i) v_{g}(j) .
\end{aligned}
$$

Assume $i \geq j$. Then $v_{f}(i) f_{i} f_{j} \geq v_{f}(j) f_{i} f_{j}$ and $v_{g}(i) g_{i} g_{j} \geq v_{g}(j) g_{i} g_{j}$, which states that a unit increase in $a_{i}(i, j)$ improves objective function by a larger quantity compared to the same amount of increase in $a_{j}(j, i)$. Considering the constraint $a_{i}(i, j)+a_{j}(j, i) \leq 1$ and 
allocation variables being nonnegative, it is concluded that $a_{i}(i, j) \geq a_{j}(j, i) \forall i \geq j$ at an optimal solution.

In fact, it is immediate to see that this result is independent from the number of agents participating in the auction and the number of distribution functions contained in $\mathcal{P}$. The interpretation is that, as expected, for a profile outcome allocating the good to the highest bidder is always more profitable if the monotone hazard condition holds. Note that when monotonicity of hazard condition fails, it is possible that the good will be allocated to a bidder with a lower valuation in the optimal mechanism. This results from the fact that the virtual valuation from a lower valuation can take a higher value than the virtual valuation under the highest bid.

Remark 1 By proof of Proposition 1, we can conclude that the optimal allocation rule obeys $a_{j}^{*}(j, i)=0 \forall(i, j) \in T^{2}$ such that $j<i$ since increasing $a_{i}(i, j)$ is always preferable to increasing $a_{j}(j, i)$ and their sum is upper bounded by 1 .

Proposition 2 If $f_{i} v_{f}(i) \geq f_{j} v_{f}(j) \forall(i, j) \in T^{2}$ such that $i \geq j \forall f \in \mathcal{P}$, the optimal allocation rule fulfills the condition $a_{i}^{*}(i, k) \geq a_{j}^{*}(j, k) \forall i \geq j$.

Proof Take arbitrary $i$ and $j$.

Case 1: $i, j<k$ then $a_{i}^{*}(i, k)=a_{j}^{*}(j, k)=0$ by Remark 1 .

Case 2: $j<k$ then $a_{i}^{*}(i, k) \geq a_{j}^{*}(j, k)=0$.

Case 3: $i, j \geq k$.

For arbitrary $i$ and $j,(14)$ and (15) can be rewritten as

$$
\begin{aligned}
& z \leq \cdots+f_{i} f_{k} a_{i}(i, k) v_{f}(i)+f_{j} f_{k} a_{j}(j, k) v_{f}(j), \\
& z \leq \cdots+g_{i} g_{k} a_{i}(i, k) v_{g}(i)+g_{j} g_{k} a_{j}(j, k) v_{g}(j) .
\end{aligned}
$$

Note that it is assumed $v_{f}(i) f_{i} \geq v_{f}(j) f_{j}$ and $v_{g}(i) g_{i} \geq v_{g}(j) g_{j}$. Since the objective function coefficient of $a_{i}(i, k)$ is higher in above equations, a unit increment in $a_{i}(i, k)$ leads to a greater improvement in objective function value than a unit increase in $a_{j}(j, k)$ would. With the fact that both $a_{i}(i, k)$ and $a_{j}(j, k)$ are bounded above by 1 (by Remark 1), the result is proved.

Although we proved Proposition 2 for two agents and two distribution functions, it is obvious that this result is valid for the general case. For the implication of Proposition 2, think of two profile outcomes where only highest bid differs and all other reported types are identical. If the seller allocates the good to the highest bidder which is the lowest in these two profile outcomes, she also sells the good in case of the second profile outcome.

Proposition 3 If $f_{i} v_{f}(i) \geq f_{j} v_{f}(j) \forall(i, j) \in T^{2}$ such that $i \geq j, \forall f \in \mathcal{P}$ and hazard function is monotone, then optimal solution ignoring monotonicity constraints is feasible to the final form of the formulation.

Proof If $f_{i} v_{f}(i) \geq f_{j} v_{f}(j) \forall(i, j) \in T^{2}$ such that $i \geq j, \forall f \in \mathcal{P}$ and hazard function is monotone, the optimal allocation rule obeys $a_{i}^{*}(i, j) \geq a_{j}^{*}(j, i)$ and $a_{i}^{*}(i, k) \geq a_{j}^{*}(j, k)$ for all $(i, j) \in T^{2}$ such that $i \geq j, \forall k \in T$ by Propositions 1 and 2 . Hence, it is directly seen that monotonicity constraints (11) are satisfied. 
Theorem 3 If all $v_{f}$ 's corresponding to $f \in \mathcal{P}$ start taking non-negative values from type $i^{*} \in T$ such that

$$
\begin{array}{ll}
v_{f}(i) \geq 0 & \forall f \in \mathcal{P}, \quad \forall i \in T \text { st. } i \geq i^{*}, \\
v_{f}(i)<0 & \forall f \in \mathcal{P}, \quad \forall i \in T \text { st. } i<i^{*},
\end{array}
$$

then optimal solution of the final formulation has the following structure:

$$
a_{i}^{*}(i, j)=\left\{\begin{array}{ll}
1 & \text { if } i \geq i^{*} \wedge i>j, \\
0.5 & \text { if } i \geq i^{*} \wedge i=j \\
0 & \text { o.w. }
\end{array} \quad \forall(i, j) \in T^{2},\right.
$$

The proof follows from the following idea. If we project out the allocation rule variables in $\left(o p t^{1}\right)$, and decompose the resulting formulation for each $f \in \mathcal{P}$ as explained before in this section, then we would obtain optimal interim allocations for each decomposed problem which are feasible with respect to given allocation rule in Theorem 3; see knapsack solution approach of Vohra (2011) for solution of decomposed subproblems. In this case, $i^{*}$ denotes the reserve price and the good is allocated with equal probability to the highest bidders if the highest bid exceeds the reserve price. To analyze the optimal structure under different circumstances, we make the following assumption. Note that this assumption does not cause loss of generality if the hazard function, and respectively, $v$ are monotone.

Assumption $1 x_{f}, x_{g} \in T$ such that $x_{f}>x_{g}$ and,

$$
\begin{gathered}
v_{f}(i) \text { is } \begin{cases}\text { nonnegative, } & \text { if } i \geq x_{f}, \\
\text { negative, } & \text { if } i<x_{f},\end{cases} \\
v_{g}(i) \text { is } \begin{cases}\text { nonnegative, } & \text { if } i \geq x_{g}, \\
\text { negative, } & \text { if } i<x_{g} .\end{cases}
\end{gathered}
$$

Assumption 1 is valid for Theorems 4, 5 and 6. We introduce the following inequality as a useful condition:

$$
\sum_{i=x_{f}}^{m} \sum_{j}^{i-1} v_{f}(i) f_{i} f_{j}+\sum_{i=x_{f}}^{m} 0.5 v_{f}(i) f_{i}^{2} \leq \sum_{i=x_{f}}^{m} \sum_{j}^{i-1} v_{g}(i) g_{i} g_{j}+\sum_{i=x_{f}}^{m} 0.5 v_{g}(i) g_{i}^{2} .
$$

Theorem 4 If condition (16) is met, the optimal solution has the following structure:

$$
a_{i}^{*}(i, j)=\left\{\begin{array}{ll}
1 & \text { if } i \geq x_{f} \wedge i>j \\
0.5 & \text { if } i \geq x_{f} \wedge i=j \\
0 & \text { o.w. }
\end{array} \quad \forall(i, j) \in T^{2},\right.
$$

Proof We aim to maximize the minimum expected revenue over distributions $f$ and $g$. Solution $a^{*}$ gives the maximum expected revenue if distribution $f$ is known to be true valuation distribution (Vohra 2011). Since maximum expected revenue with respect to $f$ is the minimum over set $\mathcal{P}$ in the case of (16), $a^{*}$ is an optimal solution.

We also need the following:

$$
\sum_{i=x_{g}}^{m} \sum_{j}^{i-1} v_{f}(i) f_{i} f_{j}+\sum_{i=x_{g}}^{m} 0.5 v_{f}(i) f_{i}^{2} \geq \sum_{i=x_{g}}^{m} \sum_{j}^{i-1} v_{g}(i) g_{i} g_{j}+\sum_{i=x_{g}}^{m} 0.5 v_{g}(i) g_{i}^{2} .
$$


Theorem 5 When condition (17) is satisfied, the optimal solution has the following form:

$$
a_{i}^{*}(i, j)=\left\{\begin{array}{ll}
1 & \text { if } i \geq x_{g} \wedge i>j, \\
0.5 & \text { if } i \geq x_{g} \wedge i=j \\
0 & \text { o.w. }
\end{array} \quad \forall(i, j) \in T^{2},\right.
$$

Proof Solution $a^{*}$ gives the maximum expected revenue for the distribution $g$ (Vohra 2011) which is the minimum in the case of (17).

Now, we propose Algorithm 1 to find the optimal solution to the robust auction design problem with ambiguity averse seller when (16) and (17) fail to hold. Algorithm 1 is instrumental in proving the structural form of the optimal auction mechanism.

During initialization, Algorithm 1 fixes $a^{*}$ for profile outcomes in which both $v_{f}$ and $v_{g}$ values of the highest bid reported are nonnegative and leads to an allocation rule that allocates the good to the highest bidders with equal probability. All other allocation variables take initial value 0 . The algorithm calculates right hand side values of (10) with the initial $a^{*}$ as $o b j_{f}$ and $o b j_{g}$. If $o b j_{f}$ is lower than or equal to $o b j_{g}$ then the algorithm stops at the current solution. The algorithm also determines $\Gamma(t)$ values for $t$ profile outcomes such that $v_{f}$ is negative but $v_{g}$ takes a value greater than or equal to 0 at the highest bid reported. If there is no such $t$ profile, the algorithm again stops at the current solution. Otherwise, at step 2, Algorithm 1 checks whether the objective value $z$ can be improved. Starting from minimum $\Gamma(t)$ value over $t$ profile outcomes as described before, the algorithm changes $a^{*}$ in such a way that the highest bid in $t$ wins the object and continues with a profile giving the next minimum $\Gamma(t)$ value until $o b j_{f}$ is equal to $o b j_{g}$ or all allocation variables are set to their upper bound (equal to 1 ) for all $t$ profiles. The procedure is clearly polynomial. The next result shows correctness of Algorithm 1.

Theorem 6 Ifneither (16) nor (17) hold, Algorithm 1 gives an optimal solution when $v_{f}(i) f_{i}$ and $\nu_{g}(i) g_{i}$ are non-decreasing in $i \in T$ and hazard function is monotone.

Proof Assume that $a^{*}$ from the algorithm violates monotonicity of interim allocations. Then $\exists i$ such that at least one of $\sum_{j=1}^{m} a_{i-1}^{*}(i-1, j) f_{j}>\sum_{j=1}^{m} a_{i}^{*}(i, j) f_{j}$ or $\sum_{j=1}^{m} a_{i-1}^{*}(i-$ $1, j) g_{j}>\sum_{j=1}^{m} a_{i}^{*}(i, j) g_{j}$ holds. Note that $f_{j}$ and $g_{j}$ are positive $\forall j \in T$. Once we prove that $a_{i}^{*}(i, j) \geq a_{i-1}^{*}(i-1, j) \forall j \in T$, this creates a contradiction.

For arbitrary $j \in T$, consider $\Gamma(i, j)$ and $\Gamma(i-1, j)$. By assumption, $v_{g}(i) g_{i} g_{j} \geq$ $v_{g}(i-1) g_{i-1} g_{j} \geq 0$ and $0 \geq v_{f}(i) f_{i} f_{j} \geq v_{f}(i-1) f_{i-1} f_{j}$. Therefore, we should have $\Gamma(i, j) \leq \Gamma(i-1, j)$. Hence, the algorithm increases $a_{i}^{*}(i, j)$ before $a_{i-1}^{*}(i-1, j)$.

If $i \neq j$,

Case 1.1: $a_{i}^{*}(i, j)=1$. Then, $a_{i}^{*}(i, j)>a_{i-1}^{*}(i-1, j)$.

Case 1.2: $a_{i}^{*}(i, j)=\frac{o b j_{f}-o b j_{g}}{v_{f}(i) f_{i} f_{j}-v_{g}(i) g_{i} g_{j}} \geq 0$. Then, the algorithm stops so that $a_{i-1}^{*}(i-$ $1, j)=0$.

Else if $i=j$,

Since $i-1<j$, the algorithm sets $a_{i-1}^{*}(i-1, j)=0$.

This proves that $a^{*}$ yields monotonic interim allocations. 


\section{Algorithm 1}

1: Initialize:

$$
\begin{aligned}
& x \leftarrow x_{f} \\
& a_{i}^{*}(i, j)=\left\{\begin{array}{ll}
1 & \text { if } i \geq x \text { and } i>j \\
0.5 & \text { if } i \geq x \text { and } i=j \\
0 & \text { ow. }
\end{array} \quad \forall(i, j) \in T^{2}\right. \\
& o b j_{f}=\sum_{i=x}^{m} \sum_{j}^{i-1} v_{f}(i) f_{i} f_{j}+\sum_{i=x}^{m}(0.5) v_{f}(i) f_{i}^{2} \\
& o b j_{g}=\sum_{i=x}^{m} \sum_{j}^{i-1} v_{g}(i) g_{i} g_{j}+\sum_{i=x}^{m}(0.5) v_{g}(i) g_{i}^{2} \\
& \Gamma=\left\{(i, j) \in T^{2} \mid x \geq i \geq x_{g} \text { and } i \geq j\right\} \\
& \Gamma(i, j)=\frac{v_{g}(i) g_{i} g_{j}}{v_{f}(i) f_{i} f_{j}} \\
& \forall(i, j) \in \Gamma
\end{aligned}
$$

2: while $\Gamma$ is not empty \& $o b j_{f}>o b j_{g}$ do

3: $\quad$ Determine $(i, j) \in \Gamma$ st. $\Gamma(i, j)=\min _{(k, l) \in \Gamma} \Gamma(k, l)$

4: $\quad$ if $i \neq j$ then

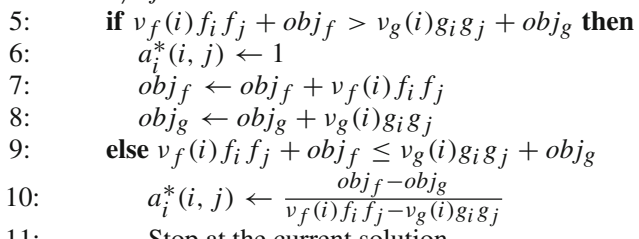

11:

12:

13:

14:

15:

16:

17:

18:

19:

20:

21:

22:

end if

end if

if $i=j$ then

$$
\begin{aligned}
& \text { if }(0.5) v_{f}(i) f_{i} f_{j}+o b j_{f}>(0.5) v_{g}(i) g_{i} g_{j}+o b j_{g} \text { then } \\
& \quad a_{i}^{*}(i, j) \leftarrow 0.5 \\
& o b j_{f} \leftarrow o b j_{f}+(0.5) v_{f}(i) f_{i} f_{j} \\
& o b j_{g} \leftarrow o b j_{g}+(0.5) v_{g}(i) g_{i} g_{j} \\
& \text { else }(0.5) v_{f}(i) f_{i} f_{j}+o b j_{f} \leq(0.5) v_{g}(i) g_{i} g_{j}+o b j_{g} \\
& \quad a_{i}^{*}(i, j) \leftarrow \frac{o b j_{f}-o b j_{g}}{(0.5) v_{f}(i) f_{i} f_{j}-(0.5) v_{g}(i) g_{i} g_{j}}
\end{aligned}
$$

Stop at the current solution.

$$
\text { end if }
$$

end if

Exclude $(i, j)$ from $\Gamma$

26: Stop at the current solution.

The algorithm considers $a_{i}(i, j)$ values only if $i \geq j$ and always assigns values between 1 and 0 . Therefore, $a^{*}$ is feasible.

Now assume that $\exists a^{\prime} \neq a^{*}$ such that it is feasible and gives $z^{\prime}>z^{*}$. Let's consider the constraint on $z$

$$
z^{*} \leq \mu_{f}^{*}+o b j_{f} \quad \forall f \in \mathcal{P},
$$


where

$$
\begin{array}{ll}
\sum_{j \in T} \sum_{i=1}^{x_{g}-1} v_{f}(i) a_{i}^{*}(i, j) \pi_{f}(i, j)=0 & \forall f \in \mathcal{P}, \\
\sum_{j \in T} \sum_{i=x_{g}}^{x_{f}-1} v_{f}(i) a_{i}^{*}(i, j) \pi_{f}(i, j)=\mu_{f}^{*} & \forall f \in \mathcal{P}, \\
\sum_{j \in T} \sum_{i=x_{f}}^{m} v_{f}(i) a_{i}^{*}(i, j) \pi_{f}(i, j)=o b j_{f} & \forall f \in \mathcal{P} .
\end{array}
$$

The point $a^{*}$ follows the structure in Theorem 3 for profiles where highest type is greater than or equal to $x_{f}$ or both reported types are less than $x_{g}$. Therefore, it is obvious that $a^{*}$ and $a^{\prime}$ are equal for these profile outcomes.

Let us first assume $a^{*}$ leads to $\mu_{f}^{*}+o b j_{f}=\mu_{g}^{*}+o b j_{g}$. Note that this is a stop condition for the algorithm. In this case, if $z^{\prime}>z^{*}$, we have the folowing:

$$
\begin{aligned}
& \mu_{f}^{\prime}+o b j_{f}>\mu_{f}^{*}+o b j_{f}, \\
& \mu_{g}^{\prime}+o b j_{g}>\mu_{g}^{*}+o b j_{g} .
\end{aligned}
$$

Then $\mu_{f}^{\prime}>\mu_{f}^{*}$ and $\mu_{g}^{\prime}>\mu_{g}^{*}$ should be satisfied so that we have:

$$
\begin{aligned}
& \mu_{f}^{*}=\sum_{j \in T} \sum_{i=x_{g}}^{x_{f}-1} v_{f}(i) a_{i}^{*}(i, j) \pi_{f}(i, j)<\sum_{j \in T} \sum_{i=x_{g}}^{x_{f}-1} v_{f}(i) a_{i}(i, j) \pi_{f}(i, j)=\mu_{f}^{\prime}, \\
& \mu_{g}^{*}=\sum_{j \in T} \sum_{i=x_{g}}^{x_{f}-1} v_{g}(i) a_{i}^{*}(i, j) \pi_{g}(i, j)<\sum_{j \in T} \sum_{i=x_{g}}^{x_{f}-1} v_{g}(i) a_{i}(i, j) \pi_{g}(i, j)=\mu_{g}^{\prime} .
\end{aligned}
$$

However, $v_{f}(i)<0$ and $v_{g}(i) \geq 0 \forall i \in T$ such that $x_{g} \leq i<x_{f}$. This creates a contradiction to $\mu_{f}^{\prime}>\mu_{f}^{*}$ and $\mu_{g}^{\prime}>\mu_{g}^{*}$.

Now assume $\mu_{f}^{*}+o b j_{f} \neq \mu_{g}^{*}+o b j_{g}$. If $\mu_{f}^{*}+o b j_{f}<\mu_{g}^{*}+o b j_{g}$, obtained solution $a^{*}$ is optimal by Theorem 4. Otherwise, $\mu_{f}^{*}+o b j_{f}>\mu_{g}^{*}+o b j_{g}$. To let $z^{\prime}>z^{*}$, we should have

$$
\mu_{g}^{\prime}+o b j_{g}>\mu_{g}^{*}+o b j_{g}
$$

This requires $\mu_{g}^{\prime}>\mu_{g}^{*}$. Note that $\Gamma$ is empty in this case. Hence, $a_{i}^{*}(i, j)$ 's $\forall(i, j) \in T^{2}$ such that $x_{g} \leq i<x_{f}$ and $i \geq j$ are at their upper bound. One can increase $a_{j}^{*}(i, j)$ values. However, this increase leads to an equal amount of decrease in corresponding $a_{i}^{*}(i, j)$ 's which have a higher opportunity cost. This creates a contradiction to existence of an optimal $a^{\prime}$ and completes the proof.

Theorem 7 For a given allocation rule $a^{*}$,

$$
p_{i}^{*}(i, j)=i a_{i}^{*}(i, j)-\sum_{k<i} a_{k}^{*}(k, j) \quad \forall(i, j) \in T^{2}
$$

is a corresponding payment rule. 
Proof Recall that in Sect. 3.2.1, we set expected payments to their tightest upper bounds:

$$
P_{f}(i)=i A_{f}(i)-\sum_{j=0}^{i-1} A_{f}(j) \quad \forall i \in T, \forall f \in \mathcal{P} .
$$

Also, by definition, we have:

$$
\begin{array}{ll}
P_{f}(i)=\sum_{j \in T} p_{i}(i, j) f_{i} f_{j} \quad \forall i \in T, \quad \forall f \in \mathcal{P}, \\
A_{f}(i)=\sum_{j \in T} a_{i}(i, j) f_{i} f_{j} \quad \forall i \in T, \quad \forall f \in \mathcal{P} .
\end{array}
$$

Now, (19) together with (20) and (21) gives (18).

We do not claim that $p^{*}$ in Theorem 7 is the unique optimal payment rule. In certain cases, it is likely to have multiple payment rules consistent with allocation rule $a^{*}$. However, $a^{*}$ is the unique optimal allocation rule as it is seen in proof of Theorem 6 . We show that there is no such $a^{\prime}$ providing a higher expected revenue to the seller but it is also clear that no other allocation rule can lead to the objective value resulting from $a^{*}$.

In the optimal mechanism, under assumptions of Theorem 6, only the highest bidder has a chance to win the object. Furthermore, an agent makes a payment only if he gets the object, and this payment does not exceed agent's type. If $x_{f}$ denotes a threshold in the optimal mechanism, for profile outcomes where the highest bid is equal to or exceeds $x_{f}$, we observe a mechanism which resembles the Vickrey auction. The highest bidder wins the object and pays an amount between the second highest bid and his own bid. When the highest bid reported is less than $x_{f}$ but bigger than or equal to $x_{g}$, for certain profile outcomes-detected by the algorithm - , the good is allocated to the highest bidder. The winner pays at most what he reported. If reported types are less than $x_{g}$, then the seller keeps the object.

On the other hand, if we relax the assumption $v_{f}(i) f_{i}$ and $v_{g}(i) g_{i}$ being non-decreasing in $i \in T$, a buyer who did not report the highest bid may have the object for certain profile outcomes. In this case, the seller makes a payment to the highest bidder.

In summary, we derived an applicable optimal mechanism for robust auction design problem. Our mechanism does not require payments higher than an agent's offer, and only the winner makes a payment to the seller, which are reasonable and fair from buyers' perspective. Moreover, the mechanism we proposed is easy to understand and it resembles the well-known Vickrey auction so that the implementation will not lead to much increased complexity.

\section{Auction design problem with ambiguity averse seller and buyers}

In this section, we investigate the case where the buyers are ambiguity averse too, in addition to the seller. The setting and the notation of the previous section apply. The objective of the problem remains identical to our setting in Sect. 3. To formulate this problem, we invoke again the Revelation Principle, and focus on direct mechanisms. 


\subsection{Formulation}

The robust auction design problem with ambiguity averse seller and buyers is formulated as follows:

$$
\begin{array}{ll}
\max _{p, a} & \left\{\min _{f \in \mathcal{P}} \sum_{i \in T} f_{i} \sum_{j \in T} p_{i}(i, j) f_{j}\right\} \\
\text { s.t. } & \min _{f \in \mathcal{P}}\left\{i \sum_{j \in T} a_{i}(i, j) f_{j}-\sum_{j \in T} p_{i}(i, j) f_{j}\right\} \geq 0 \quad \forall i \in T \\
& \min _{f \in \mathcal{P}}\left\{i \sum_{k \in T} a_{i}(i, k) f_{k}-\sum_{k \in T} p_{i}(i, k) f_{k}\right\} \geq, \\
& \min _{f \in \mathcal{P}}\left\{i \sum_{k \in T} a_{j}(j, k) f_{k}-\sum_{k \in T} p_{j}(j, k) f_{k}\right\} \quad \forall i, j \in T, \\
& \sum_{i \in T} n_{i}(t) a_{i}(t) \leq 1 \quad \forall t \in T^{2} \\
& a_{i}(i, j) \geq 0 \quad \forall i, j \in T \\
& p_{i}(i, j) \geq 0 \quad \forall i, j \in T .
\end{array}
$$

For ease of notation, we give the formulation for the case where there are two agents. Individual Rationality constraints (23) ensure that each agent gains at least zero payoff from participation. Incentive Compatibility constraints, which force agents to truthfully report their types, are given in (24) because the bidders consider the worst case payoffs due to ambiguity aversion. This model is reformulated as the following Mixed Integer Programming (MIP) Problem:

$$
\begin{aligned}
& \max _{z, p, a, D, b} \quad z \\
& \text { s.t. } \quad z \leq \sum_{i \in T} f_{i} \sum_{j \in T} p_{i}(i, j) f_{j} \\
& i \sum_{j \in T} a_{i}(i, j) f_{j}-\sum_{j \in T} p_{i}(i, j) f_{j} \geq 0 \quad \forall f \in \mathcal{P}, \forall i \in T, \\
& D_{i j} \leq i \sum_{k \in T} a_{j}(j, k) f_{k}-\sum_{k \in T} p_{j}(j, k) f_{k} \quad \forall f \in \mathcal{P}, \forall i, j \in T, \\
& D_{i j}+M b_{f}(i, j) \geq \text {, } \\
& i \sum_{k \in T} a_{j}(j, k) f_{k}-\sum_{k \in T} p_{j}(j, k) f_{k} \quad \forall f \in \mathcal{P}, \forall i, j \in T \text {, } \\
& \sum_{f \in \mathcal{P}} b_{f}(i, j) \leq|\mathcal{P}|-1 \quad \forall i, j \in T, \\
& i \sum_{k \in T} a_{i}(i, k) f_{k}-\sum_{k \in T} p_{i}(i, k) f_{k} \geq D_{i j} \quad \forall f \in \mathcal{P}, \forall i, j \in T, \\
& b_{f}(i, j) \in\{0,1\} \quad \forall f \in \mathcal{P}, \forall i, j \in T,
\end{aligned}
$$

where $M$ is a sufficiently large number, and (25)-(27) hold.

We introduced the dummy variables $D_{i j}$ which take the value of minimum expected payoff over $f \in \mathcal{P}$ if an agent with true valuation $i$ reports $j$ by (30), (31) and (32). The number 
of distributions in $\mathcal{P}$ leads to a considerable increase in the number of constraints, which contributes to the difficulty of the problem. Furthermore, the optimal solution does not seem to give a recognizable auction mechanism. Next, we introduce a numerical algorithm which enables to achieve an optimal solution with a reduced version of the formulation.

\subsection{A hybrid algorithm}

Assume $\mathcal{P}=\left\{f^{0}, f^{1}, \ldots, f^{m}\right\}$. Let $\mathcal{P}^{\prime}$ be a subset of $\mathcal{P}$ and $\operatorname{MIP}\left(\mathcal{P}^{\prime}\right)$ is a reformulation of MIP in which set $\mathcal{P}$ is replaced by $\mathcal{P}^{\prime}$. In other words, we take a subset of distributions in $\mathcal{P}$ and eliminate constraints and variables corresponding to remaining distributions.

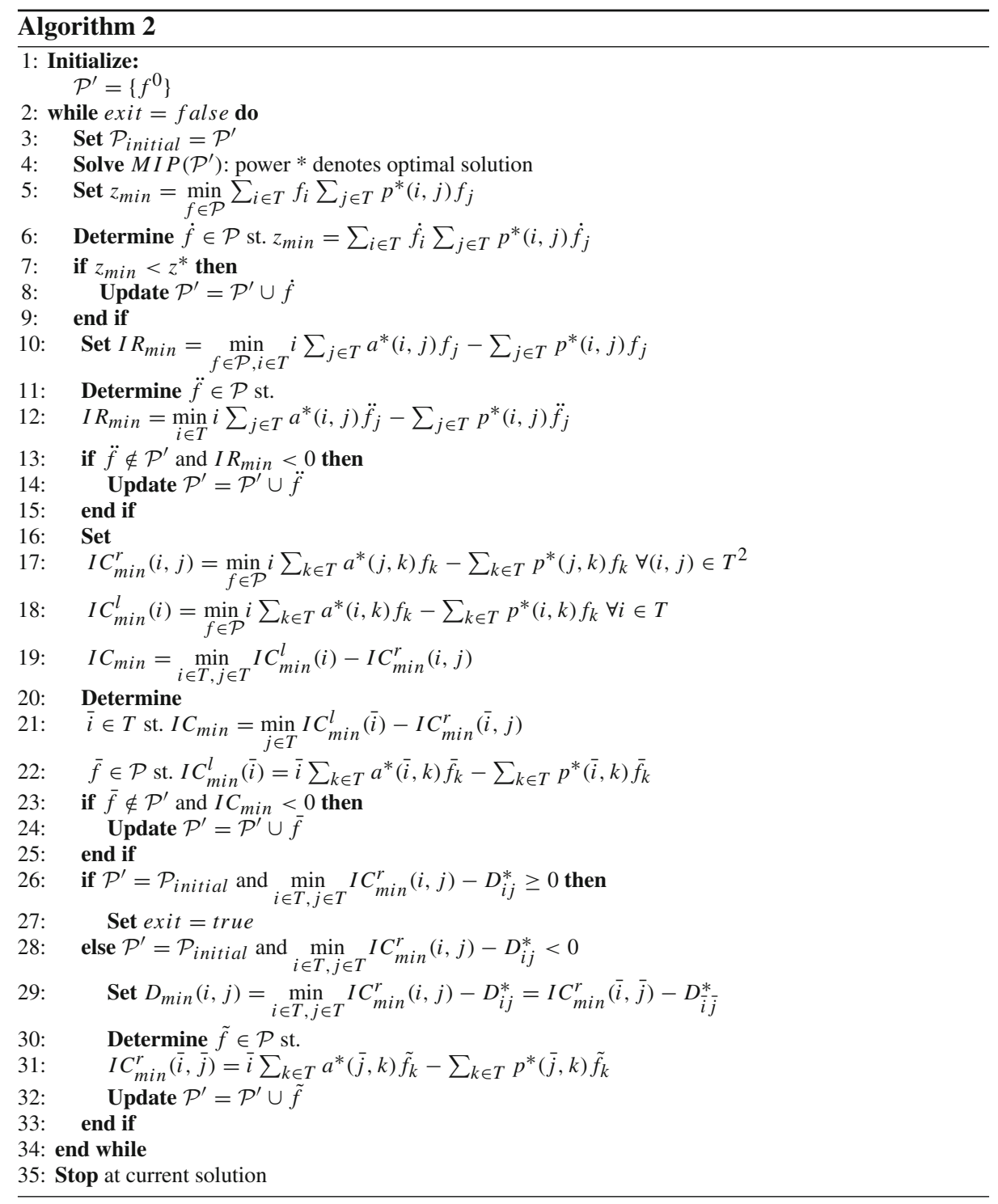


According to our computational study, Algorithm 2 obtains very accurate solutions to the MIP formulation. The algorithm starts by solving MIP only over one distribution function, $f^{0}$. Using the optimal solution obtained, rows 5-9 check whether the constraint type (28) is satisfied by remaining distribution functions in $\mathcal{P}$ and determine the most violated one. The distribution function which causes the most violated constraint is added to $\mathcal{P}^{\prime}$. Violation in Individual Rationality constraints (29) is detected in 10-15. Again, detected distribution function is added to $\mathcal{P}^{\prime}$ if it is not already in it. Note that it is possible to observe identical distribution functions from 5 to 9 and 10 to 15 .

The algorithm does not consider constraints (30) to (32) in MIP. The reason is that it also updates the right hand side of constraint (32) according to $\mathcal{P}^{\prime}$. This corresponds to the fact that $D_{i j}$ 's now take the value of minimum expected payoff over $f \in \mathcal{P}^{\prime}$ instead of $f \in \mathcal{P}$ if an agent with true valuation $i$ reports $j$. This causes a restriction rather than a relaxation.

From row 16 to row 25, the algorithm detects violation in constraint (33). However, the algorithm does not utilize the $D_{i j}$ 's for the right-hand side as explained above. The algorithm calculates the right hand side of each constraint as $I C_{\text {min }}^{r}(i, j)$, the minimum expected payoff with observed optimal solution values over $f \in \mathcal{P}$ if an agent with true valuation $i$ reports $j$. Using these right hand side values, the most violated constraint is determined, and the corresponding distribution function is included in $\mathcal{P}^{\prime}$.

If at least one distribution function is added to $\mathcal{P}^{\prime}$, the algorithm repeats the process starting from row 3. If $\mathcal{P}^{\prime}$ remains the same, it is concluded that observed solution is feasible to original formulation. Since the algorithm also restricts the problem, it may not be optimal. Therefore, this restriction is questioned by looking at the difference between $D_{i j}^{*}$ and $I C_{\text {min }}^{r}(i, j)$ for all $(i, j) \in T^{2}$. The distribution function causing the highest difference is added to $\mathcal{P}^{\prime}$ and the process is repeated from 3 until no restriction or violation is detected.

In each step, the algorithm gives a bound to the optimal value of the problem. However, it is hard to determine if it is a lower or an upper bound because some constraints of MIP formulation are relaxed while some are restricted. On the other hand, under certain conditions we can say more about the bound observed. If $\mathcal{P}^{\prime}$ remains unchanged until row 25 , the previously observed solution is feasible to MIP. Therefore, it is a lower bound for the problem.

The algorithm can be adjusted to obtain an upper bound for MIP formulation. If $|\mathcal{P}|-1$ in constraint (32) is not updated depending on $\mathcal{P}^{\prime}$ and remains the same throughout the algorithm, then we observe an upper bound in each step.

\subsection{Computational results}

We have two buyers in all instances reported in this section.

In Tables 1 and 2, each row corresponds to one problem instance. Solution times are given in seconds. $|\mathcal{P}|$ is the total number of distributions in set $\mathcal{P}$ and $|T|$ is the number of valuations. Iterations column shows how many times the while loop in the algorithm was executed to obtain the solution. $\left|P^{\prime}\right|$ represents the final number of distributions in set $\mathcal{P}^{\prime}$ when the algorithm stops. $\mathcal{P}$ sets belonging to instances grouped in double lines are randomly generated within an $\left(f^{0} \pm \epsilon\right)$ interval from the same given $f^{0}$ and $\epsilon$. While randomizing input data, we ensure that distribution values do not take negative values. Input data can be obtained from the authors upon request. We used NetBeans IDE 8.0.1 and CPLEX Studio 12.6.1 for solving all instances. In all rows, we give the optimal objective value (or the best objective function value within allotted time if applicable) and total solution time for MIP. However, for the values written in italic, as in row 14, we set a time limit and observed the best integer solution within the allotted time. The idea was to see whether the algorithm is capable of obtaining an optimal solution within 15 min of computing time while the state-of-the-art 
Table 1 Numerical results 1

\begin{tabular}{|c|c|c|c|c|c|c|c|c|}
\hline \multirow{2}{*}{\multicolumn{2}{|c|}{$|\mathcal{P}|$}} & \multirow[t]{2}{*}{$|T|$} & \multicolumn{2}{|l|}{ MIP formulation } & \multicolumn{4}{|l|}{ Algorithm } \\
\hline & & & Objective value & Solution time & Objective value & Solution time & Iterations & $\left|P^{\prime}\right|$ \\
\hline 1 & 20 & 5 & 1.293066 & 17.914 & 1.293066 & 10.583 & 7 & 12 \\
\hline 2 & 20 & 5 & 1.290019 & 23.493 & 1.290019 & 5.304 & 6 & 10 \\
\hline 3 & 20 & 5 & 1.281526 & 34.728 & 1.281526 & 9.345 & 6 & 10 \\
\hline 4 & 20 & 5 & 1.22677 & 22.275 & 1.22677 & 15.883 & 8 & 12 \\
\hline 5 & 20 & 5 & 1.223931 & 25.897 & 1.223931 & 28.167 & 9 & 13 \\
\hline 6 & 20 & 5 & 1.222235 & 51.394 & 1.222235 & 2.559 & 5 & 7 \\
\hline 7 & 20 & 5 & 1.346207 & 8.332 & 1.346207 & 4.289 & 5 & 10 \\
\hline 8 & 20 & 5 & 1.304475 & 52.233 & 1.304475 & 9.357 & 7 & 11 \\
\hline 9 & 20 & 5 & 1.293792 & 9.43 & 1.293792 & 7.221 & 6 & 10 \\
\hline 10 & 20 & 6 & 1.714115 & 884.722 & 1.714115 & 970.904 & 10 & 18 \\
\hline 11 & 20 & 6 & 1.75066 & 919.362 & 1.75066 & 112.23 & 8 & 15 \\
\hline 12 & 20 & 6 & 1.724949 & 1535.607 & 1.724949 & 788.868 & 11 & 15 \\
\hline 13 & 20 & 6 & 1.637257 & 6070.151 & 1.637257 & 596.016 & 7 & 11 \\
\hline 14 & 20 & 6 & 1.672065 & 900.02 & 1.672065 & 142.404 & 6 & 11 \\
\hline 15 & 20 & 6 & 1.661494 & 900.02 & 1.661494 & 255.527 & 7 & 11 \\
\hline 16 & 20 & 6 & 1.076266 & 375.062 & 1.076266 & 318.536 & 11 & 13 \\
\hline 17 & 20 & 6 & 1.082449 & 518.958 & 1.082449 & 462.247 & 13 & 16 \\
\hline 18 & 20 & 6 & 1.089408 & 408.33 & 1.089408 & 164.907 & 10 & 12 \\
\hline 19 & 20 & 6 & 1.124627 & 900.03 & 1.124869 & 663.018 & 8 & 12 \\
\hline 20 & 20 & 6 & 1.10295 & 900.02 & 1.10295 & 102.121 & 6 & 10 \\
\hline 21 & 20 & 6 & 1.116765 & 900.02 & 1.116765 & 453.76 & 9 & 13 \\
\hline
\end{tabular}

MIP solver runs into the time limit. Indeed, for all five instances where the MIP solver stops because of time limit, our algorithm produced the optimal solution in considerably shorter time.

From Tables 1 and 2, we see that the hybrid algorithm for the robust auction design problem leads to significant time efficiency, and obtains optimal solution for all instances. We note some minor differences between objective values from MIP formulation and the algorithm in rows 24, 32 and 40 due to tolerances in Java Programming Language.

Increasing number of iterations and the expanding cardinality of $\mathcal{P}^{\prime}$ have a marked effect on the improvement that our algorithm brings. Consider instances 4-6. For solving the instance reported in row 6 , the number of iterations is equal to 5 , and the final number of distributions included in $\mathcal{P}^{\prime}$ is 7 , when compared to instances 4 and 5 , this is very low. Hence, as expected, the reduction in solution time by the algorithm is noticeably higher than in rows 4 and 5 both in percentage and net amount.

Total solution times seem to depend on all randomized distributions in $\mathcal{P}$ rather than only given $f^{0}$ and $\epsilon$. For example, although $\mathcal{P}$ sets corresponding to instances 34-36 are randomized in a similar fashion, instance 36 has a huge solution time compared to others. This difference is reduced for our algorithm solution time even though the number of iterations and $\left|\mathcal{P}^{\prime}\right|$ values of 36 are not the lowest in this sample. This tells us that the number of iterations and the final number of distributions are not the only elements determining the time efficiency brought about by the algorithm. 
Table 2 Numerical results 2

\begin{tabular}{|c|c|c|c|c|c|c|c|c|}
\hline \multirow{2}{*}{\multicolumn{2}{|c|}{$|\mathcal{P}|$}} & \multirow[t]{2}{*}{$|T|$} & \multicolumn{2}{|l|}{ MIP formulation } & \multicolumn{4}{|l|}{ Algorithm } \\
\hline & & & Objective value & Solution time & Objective value & Solution time & Iterations & $\left|P^{\prime}\right|$ \\
\hline 22 & 20 & 6 & 1.064647 & 263.756 & 1.064647 & 149.095 & 8 & 13 \\
\hline 23 & 20 & 6 & 1.078787 & 67.818 & 1.078787 & 34.016 & 7 & 11 \\
\hline 24 & 20 & 6 & 1.073474 & 156.101 & 1.073538 & 81.204 & 8 & 13 \\
\hline 25 & 15 & 6 & 1.092282 & 835.478 & 1.092282 & 28.419 & 6 & 9 \\
\hline 26 & 15 & 6 & 1.105905 & 196.086 & 1.105905 & 101.928 & 6 & 10 \\
\hline 27 & 15 & 6 & 1.092442 & 59.869 & 1.092442 & 22.295 & 5 & 8 \\
\hline 28 & 15 & 6 & 1.103278 & 381.92 & 1.103278 & 25.897 & 6 & 8 \\
\hline 29 & 15 & 6 & 1.114296 & 332.558 & 1.114296 & 170.605 & 8 & 11 \\
\hline 30 & 15 & 6 & 1.111806 & 238.398 & 1.111806 & 335.704 & 8 & 11 \\
\hline 31 & 15 & 6 & 1.084282 & 78.431 & 1.084282 & 51.805 & 7 & 11 \\
\hline 32 & 15 & 6 & 1.041195 & 142.567 & 1.041202 & 83.884 & 9 & 11 \\
\hline 33 & 15 & 6 & 1.050686 & 157.032 & 1.050686 & 78.218 & 7 & 9 \\
\hline 34 & 10 & 7 & 0.765615 & 95.12 & 0.765615 & 10.727 & 3 & 5 \\
\hline 35 & 10 & 7 & 0.746313 & 70.392 & 0.746313 & 141.371 & 5 & 6 \\
\hline 36 & 10 & 7 & 0.777228 & 14263.43 & 0.777228 & 120.816 & 4 & 6 \\
\hline 37 & 10 & 7 & 1.052553 & 33.193 & 1.052553 & 75.399 & 6 & 9 \\
\hline 38 & 10 & 7 & 1.070767 & 960.996 & 1.070767 & 417.991 & 6 & 8 \\
\hline 39 & 10 & 7 & 1.085262 & 227.791 & 1.085262 & 74.282 & 6 & 8 \\
\hline 40 & 10 & 7 & 1.027729 & 156.642 & 1.027727 & 92.235 & 5 & 8 \\
\hline 41 & 10 & 7 & 1.065472 & 209.731 & 1.065472 & 34.3 & 6 & 6 \\
\hline 42 & 10 & 7 & 1.04994 & 226.327 & 1.04994 & 223.999 & 6 & 8 \\
\hline
\end{tabular}

While we cannot make a precise conclusion on the class of instances for which the algorithm will be efficient, we can conclude that the algorithm effectively reduces the solution time in most cases.

\section{Conclusion}

In this paper we focused on the auction design problem with discrete valuations for a single good when buyers' valuation distribution comes from a set of distributions $\mathcal{P}$ rather than being unique and known to all parties. We assumed both the seller and the buyers are risk neutral. In Sect. 3, we gave a formulation for robust auction design problem with an ambiguity averse seller and $n$ ambiguity neutral buyers. Then, we reformulated the problem with the help of standard results from linear programming. We derived the structure of the optimal solution for the case where there are two buyers and $\mathcal{P}$ consists two discrete distributions under certain assumptions. For the case where the assumptions do not hold, we gave an algorithm to determine the optimal auction mechanism. In the optimal mechanism, the highest bidders win the object with equal probability until the highest bid reported falls under a threshold. Only the winner makes a payment and he pays an amount between his own bid and second highest bid. Under the threshold, there may be allocation to the highest bid for some profile outcomes and these are determined by the algorithm. Although there have been studies in 
the literature underlying few properties of the optimal mechanism to robust auction design problem (Bose et al. 2006), a specific mechanism was not given, to the best of our knowledge. The optimal mechanism we derived is both detailed and applicable. It is easy to understand because it resembles the well-known Vickrey auction and it does not require payments which exceed the buyer's offer. Furthermore, only the winner makes a payment, which is reasonable and fair from buyers' perspective. Hence, the implementation of our study will not lead to much increased difficulty of implementation.

In Sect. 4, we analyzed the same problem when the buyers are also ambiguity averse. This problem is known to be very complex, and consequently the literature is very limited (Bose et al. 2006). We formulated the problem as a mixed integer programming problem, and to the best of our knowledge our formulation is novel. Then, we proposed an algorithm which enables to solve the problem more efficiently than state-of-the-art general purpose MIP solvers. Our computational results show that the algorithm leads to time efficiency, and computes an accurate solution for the instances considered.

There are several research directions arising from our study. In Sect. 3, while deriving an optimal mechanism, we assumed that there are two distinct distributions in set $\mathcal{P}$. Perhaps, under certain assumptions, it might be possible to consider other forms for the set $\mathcal{P}$. The effect of additional constraints such as budget constraints on the optimal mechanism can also be considered as future work.

\section{References}

Bandi, C., \& Bertsimas, D. (2014). Optimal design for multi-item auctions: A robust optimization approach. Mathematics of Operations Research, 39(4), 1012-1038.

Bose, S., Ozdenoren, E., \& Pape, A. (2006). Optimal auctions with ambiguity. Theoretical Economics, 1(4), 411-438.

Ellsberg, D. (1961). Risk, ambiguity, and the savage axioms. The Quarterly Journal of Economics, 75(4), 643-669.

Gilboa, I., \& Schmeidler, D. (1989). Maxmin expected utility with non-unique prior. Journal of Mathematical Economics, 18(2), 141-153.

Hurwicz, L., \& Reiter, S. (2006). Designing economic mechanisms. Cambridge: Cambridge University Press. Klemperer, P. (1999). Auction theory: A guide to the literature. Journal of Economic Surveys, 13(3), 227-286.

Krishna, V. (2009). Auction theory. London: Academic Press.

Lo, K. C. (1998). Sealed bid auctions with uncertainty averse bidders. Economic Theory, 12(1), 1-20.

Myerson, R. B. (1981). Optimal auction design. Mathematics of Operations Research, 6(1), 58-73.

Riley, J. G., \& Samuelson, W. F. (1981). Optimal auctions. The American Economic Review, 71(4), 381-392.

Salo, A., \& Weber, M. (1995). Ambiguity aversion in first-price sealed-bid auctions. Journal of Risk and Uncertainty, 11(2), 123-137.

Vickrey, W. (1961). Counterspeculation, auctions, and competitive sealed tenders. The Journal of Finance, $16(1), 8-37$.

Vohra, R. V. (2011). Mechanism design: A linear programming approach (Vol. 47). Cambridge: Cambridge University Press.

Vohra, R. V. (2012). Optimization and mechanism design. Mathematical Programming, 134(1), $283-303$. 